\title{
The effect of manual acupuncture and electroacupuncture on lower limb muscle strength
}

\section{温针炎治疗勃起功能障碍的临床疗效观察}

\author{
Stephen Payton ${ }^{1}$, Steven D Bailey ${ }^{2}$ \\ 1 Teesside University, Middlesbrough TS1 3BX, UK \\ 2 School of Biomechanics Sports and Remedial Therapies, Nottingham NG10 4LB, UK
}

\begin{abstract}
Objective: To determine whether six weeks' of acupuncture has a positive effect on plantar flexion muscle strength and whether electroacupuncture (EA) has a greater effect than manual acupuncture (MA) on plantar flexion, so that the most beneficial method of acupuncture can be established for strengthening muscles.

Methods: A randomized controlled trial with parallel groups. A total of 20 participants were randomly assigned to three groups: control/non-acupuncture group $(n=6)$, manual acupuncture group (MAG) $(n=8)$ and electroacupuncture group (EAG) $(n=6)$. MAG and EAG received three 25 minute treatment sessions per week for 6 weeks. The control group continued with their normal training activity and received no treatment. The maximal voluntary contraction (MVC) of the ankle plantar-flexors at $30^{\circ}$ per second was measured using Biodex system 3.

Results: Both MA and EA had a positive effect in increasing plantar flexion strength. The likelihood for this happening was high; 69\% for MA and 92\% for EA when compared with the control group. However the effect size was small for both groups. There was only a trivial difference in the effect size between the EAG and the MAG in regards to average plantar flexion torque.

Conclusion: EA and MA have a beneficial effect on plantar flexion muscle strength compared with the control group in healthy population.

Keywords: Acupuncture Therapy; Electroacupuncture; Manual Acupuncture; Muscle Strength; Muscle Strength Dynamometer; Randomized Controlled Trial

【摘要】目的: 验证 6 星期针刺治疗对跖屈肌肌力是否有积极影响, 并比较电针和手捻针的效果, 从而确立增强肌 力的最佳针刺方法。方法: 研究采用随机平行对照法。20 例参与者随机分为 3 组: 对照/非针刺组 $(n=6)$, 手捻针组 $(n=8)$ 及电针组 $(n=6)$ 。手捻针组及电针组每星期各治疗 3 次, 1 次 $25 \mathrm{~min}$, 共治疗 6 星期。对照组仅予以一般训练。 应用 Biodex system 3 测量踝关节跖屈位 $30^{\circ}$ 时跖屈肌每秒最大自主收缩力。结果: 手捻针与电针皆可提高跖屈肌肌 力, 与对照组比较, 起效可能性都较高: 手捻针为 $69 \%$, 电针为 $92 \%$ 。但两个针刺组的效应量都较小。两个针刺组平 均跖屈力矩比较, 效应量差异不显著。结论: 对于健康人群, 电针及手捻针都有利于提高跖屈肌力量。
\end{abstract}

【关键词】针刺疗法; 电针; 手捻针; 肌力; 测力计; 随机对照试验

【中图分类号】R246.2【文献标志码】A

Acupuncture is widely used in sport for its analgesic effect on a variety of joints ${ }^{[1-2]}$. More recently acupuncture has been used to enhance recovery from sporting competitions ${ }^{[3]}$. Recently there has been a broader acceptance of acupuncture aimed at enhancing sports performance. Acupuncture point Zusanli (ST 36) is widely accepted ended under traditional Chinese medicine (TCM) as a point to develop strength. Over the past two decades there have been a number of studies $^{[4-12]}$ that have shown positive results with

Author: Stephen Payton, MSc Sport Therapy, BSc Sport Science.

E-mail: s.payton@tees.ac.uk

regards to the effects of acupuncture on enhancing psychological and physiological responses in athletes during and following intense exercise. The four main areas that acupuncture is utilized in enhancing human performance during or following exercise include improving muscle strength, reducing delayed onset of muscle soreness (DOMS), reducing lactic acid, and heart rate reduction following exercise ${ }^{[13]}$. Muscle strength is an integral part of an athletes training programme for injury prevention, rehabilitation following injury and in order to enhance performance in preparation for competition. Strength training has been shown to have a significant impact on a variety of sports ${ }^{[9,11-12,14]}$ and ultimately be an effective ergogenic aid in the enhancement of muscle strength of the lower limb. A systematic review undertaken by Bailey ${ }^{[13]}$ to investigate the effectiveness of acupuncture in enhancing human 
performance determined that methodological quality and/or the STRICTA (Standards for Reporting Interventions in Controlled Trials of Acupuncture) of these studies have been shown to be of poor quality. The results from the finding of these studies suggest that there is a neural underlying mechanism involved in enhancing muscle strength ${ }^{[15]}$. The aim of this study is to determine the effect of acupuncture on ankle plantar flexion muscle strength and to discuss the underlying neural and non-neural mechanism of acupuncture on enhancing muscle strength.

\section{Methods}

\subsection{Participants}

Participants were volunteers recruited via posters and email advertisement. Ethics was obtained through Teesside University Ethics Committee for the School of Social Science Business and Law.

\subsection{Inclusion criteria}

Subjects will not currently have or were not recently recovering from a lower limb musculoskeletal injury; able to provide informed written consent; no previous experience of acupuncture.

\subsection{Exclusion criteria}

Needles phobia; under the age of 18 years; under supervision of another health practitioner; on medication for musculoskeletal (MSK) injury; blood donor; impaired sensation; any neurological disorder within the last 6 months; pregnant.

Upon recruitment and prior to any testing, participants were given a full explanation of the study along with a patient information sheet. Participants were then asked to complete an informed written consent form and a medical questionnaire.

\subsection{Randomization}

Participants were randomly assigned using the minimization technique Hopkins ${ }^{[16]}$, to one of three groups: a control group, a manual acupuncture group (MAG) and an electroacupuncture group (EAG).

\subsection{Interventions}

The study design is a randomized controlled parallel group trial. All participants completed a familiarization sessions before pre-tests in order to reduce learning effect. Pre-tests were used to determine baseline maximal voluntary contraction (MVC) of the ankle plantar flexor muscles. A post-test was carried out after 6 weeks of acupuncture treatment in order to determine any change in MVC of the ankle plantar flexor. All participants strength testing was conducted using a Biodex System 3 situated at Teesside University (HS 1.03). The seating position, knee and hip joint angles were all recorded during the familiarization session and used during the pre- and post-test to eliminate differences in strength output due to seating position. The lower limbs were strapped in place using a Biodex binding system and the upper body was strapped in using a harness to eliminate upper body recruitment to facilitate the movement. The contraction force was shown on a computer monitor, which was linked to the Biodex dynamometer to provide visual feedback. In each testing session, each group performed five sets of voluntary concentric contractions, at $30 \%$ with $90 \mathrm{~s}$ recovery between each repetition. For each repetition, the participants were asked to apply maximal force through the full range of movement.

In this study a Biodex system 3 was used to measure torque and power. The Biodex has been found to be highly reliable ${ }^{[17]}$ in re-test situations. The rate of contraction at $30 \%$ was chosen to assess ankle plantar flexion muscle strength as maximal strength is elicited during slow range of movements less than $45^{\circ}$ per second and the lower limb tricep surae calf complex is composed primarily of slow twitch muscle.

Fast twitch and faster speeds are an assessment of power. Type I slow twitch muscle fiber has a long twitch time and limited potential for rapid force development ${ }^{[18]}$. Furthermore higher forces are generated at lower speeds ${ }^{[19]}$. A recent study by Hauraix ${ }^{[20]}$ found that force of the plantar flexor muscles is significantly higher at $30 \%$ compared with other velocities. Therefore the rate of 300/s was chosen due to the nature of the muscle type and previous literature. Thus utilizing a slow speed of $3030 \%$ will quantify any changes in strength.

\subsubsection{Reason for point selection}

A number of studies ${ }^{[9-14]}$ have demonstrated the effects of Zusanli (ST 36), Xiajuxu (ST 39) and Sanyinjiao (SP 6) in enhancing muscle strength in the lower limb. Jarmey and Bouratinos ${ }^{[21]}$ state that Zusanli (ST 36) can be stimulated on athletes before and after exercising in order to strengthen the legs, in support of this ${ }^{[22]}$ state that Zusanli (ST 36) is one of the great points in energy giving. When giving stimulating massage it provides a general energy tonic and can be used in energizing muscles and enhancing performance. Acupoints Xiajuxu (ST 39) and Sanyinjiao (SP 6) are common points used for weakness and atrophy disorders of the lower limb.

Mr Stephen Payton a qualified sports therapist who had undertaken post-graduate training in integrated Western and classical Chinese acupuncture with two years' postgraduate acupuncture experience, performed the acupuncture. He was closely guided by a colleague who has more than 10 years' experience and a master's degree in Acupuncture.

\subsubsection{Manual acupuncture (MA) procedure}

Zusanli (ST 36), Xiajuxu (ST 39) and Sanyinjiao (SP 6) were needled using disposable sterile single use Phoenix C-type needles $(0.25 \mathrm{~mm}$ in diameter and 40 $\mathrm{mm}$ in length). Needles were inserted perpendicularly to a depth of 10-15 mm depending on the subjects' constitution (e.g. skin thickness, subcutaneous fat layer 
thickness) and manipulated (bi-directionally rotated) until qi arrival (i.e. needle sensation, a soreness and numbness sensation but not a sharp pain) was reported then left in place for $25 \mathrm{~min}$. The needles were then manipulated (bi-directionally rotated) every 5 min for 15 s. All participants were advised to maintain the same lifestyle for duration of the course of the study. All participants in the MAG visited the laboratory three times per week for six weeks.

\subsubsection{EA procedure}

Zusanli (ST 36), Xiajuxu (ST 39) and Sanyinjiao (SP 6) were needled using disposable sterile single use Phoenix C-type needles $(0.25 \mathrm{~mm}$ in diameter and 40 $\mathrm{mm}$ in length). Needles were inserted perpendicularly to a depth of 10-15 mm depending on the subjects' constitution and manipulated (bi-directionally rotated) until qi arrival was reported. Then electrodes from a transcutaneous electrical nerve stimulation (TENS) machine, model TPN 200 easy (Everyway Instruments Co., Ltd., Taiwan, China), was attached to the needles, delivering a constant current share wave form at a frequency of $120 \mathrm{~Hz}$ pulsed width $1 \mathrm{~ms}$ and the intensity up to the maximum level the subject could tolerate for $25 \mathrm{~min}$. A frequency of $120 \mathrm{~Hz}$ was chosen as the report by Maffiuletti NA, et $a l^{[23]}$ demonstrated strength gain in knee extensor and plantar flexor muscle MCV using electro-stimulation at $115-120 \mathrm{~Hz}$. Furthermore, Blu ${ }^{[24]}$ states that a complete tetanus in skeletal muscle will be reached between a frequency of $50 \mathrm{~Hz}$ and $200 \mathrm{~Hz}$. Participants were instructed not to voluntarily contract the muscle in either limb during the session. All participants were advised to maintain same lifestyle for duration of the course of the study. All participants in the EAG visited the laboratory three times per week for six weeks.

\subsubsection{Control procedure}

The participants in all three groups continued with their normal daily activities without engaging in any specific physical training. All participants including the control group visited the clinic. The control group rested on a plinth in the same position as the other participants in the other two groups but did not receive acupuncture to reduce the potential effects from the experimental environment. All participants in the control group visited the laboratory three times per week for six weeks. All participants were advised to maintain the same lifestyle for the duration of the course of the study.

\subsection{Data analysis}

For tests of main effects between groups, an analysis of co-variance (ANCOVA) with Sidak comparison and adjustments for sex, age and muscle strength of ankle plantar flexion scores at baseline was utilized.

Effect sizes from baseline for each group were calculated with the following formula: Cohen's $d=M 1$ $-\mathrm{M} 2 \div[(\mathrm{SD} 1+\mathrm{SD} 2) \div 2]$. Cohen's measure $\mathrm{d}=0.20$ is considered a small effect, $d=0.50$ is a moderate effect and $d=0.80$-infinity is a large effect size ${ }^{[25-26]}$.

All analyses were performed using the analysis of a pre-post parallel-groups controlled trial with adjustment for a predictor spreadsheet ${ }^{[27]}$. This sheet enables the use of the pre-test score as a covariate to control for baseline imbalances in our measures between the control and the acupuncture groups ${ }^{[28]}$. This sheet also allows for the magnitude based inferences approach to obtain a more practical and pivotal observation of the effect of acupuncture. Previous research has focused on probabilities and determining statistical significance or non-significant on the basis of a $\mathrm{P}$ value, quite often 0.05 , which only tells us there is less than one in 20 chance of being wrong; nothing about what effect the intervention has on the participants. This approach of focusing on the $P$ value results in confusion and can be misleading, depending on the magnitude of the statistic, error of measurement, and sample size ${ }^{[29]}$. Hopkins ${ }^{[26]}$ and Cohen ${ }^{[25]}$ based inference statistics provide information on the basis of the magnitude of the effect the intervention is having. Statistical significance is concerned with whether a research result is due to chance or sampling variability; practical significance is concerned with whether the result is useful in the real world ${ }^{[30]}$. For this reason, this paper will report results as effect size and statistical significance. The thresholds of the clinical inference within the results which distinguishes positive and negative values have been set for the minimum chance of benefit $25 \%$ and the maximum risk of harm $0.5 \%$ resulting.

\section{Results}

All participants completed the study with no dropouts and no adverse events reported by any of the participants.

An anova was performed on baseline data before the magnitude inference statistics were performed to determine whether there were any differences between groups at the start of the study. No significant differences were found in subject characteristics and baseline values of average peak torque in ankle plantar flexion of the right and left foot between the EAG, MAG and control group at the start of the study.

\subsection{Average torque ankle plantar-flexion}

Table 1 shows the likelihood, effect size and clinical implications. It clearly shows MA to be more beneficial than the control group after 6 weeks' treatment. An effect size for the right foot average torque ankle plantar flexion was $(0.27 \pm 0.26)$ (small Cohen), with a likelihood of $69 \%(69 / 31 / 0)$ chance that the intervention will have a beneficial effect in the population. The effect size of the left ankle average torque value for plantar flexion was $(0.51 \pm 0.42)$ (moderate), with a likelihood of (89/10/1), an $89 \%$ chance that the intervention will have 
a beneficial effect which is higher than the right foot. The mechanistic effect is positive. The large effect and higher likelihood in the left limb is likely to be due to the nature on the non-dominant limb responding better to the intervention.

Table 2 shows the likelihood and effect size to be significantly more beneficial in the EA group than the control group after 6 weeks. An effect size for right foot average torque ankle plantar flexion was $(0.4 \pm 0.24)$ (small), with a likelihood of $(92 / 8 / 0)$, thus there is a $92 \%$ chance that the intervention will have a beneficial effect. The left ankle average torque plantar flexion has a greater effect size of $(0.64 \pm 0.4)$ (medium), with a likelihood of (96/4/0), a 96\% chance. The data show again that acupuncture has had a positive effect on strength. The results also show that the likelihood of a positive response is higher with EA compared to MA. Furthermore, the EA results show a higher response to the intervention in the left lower limb with regards to both strength and likelihood of the response.

The data for the right foot show that EA versus MA $(-0.17 \pm 0.32)$ is trivial in effect size $(<0.2$ considered trivial according to Cohen 1992). This indicates that there is no clinical difference between the two treatment groups for the right foot. However, the data for the left lower limb show that it is possible that the effect of EA is slightly larger than that of MA $(-0.34 \pm$ 0.62 ) but the SD is large and the likelihood has a wide spread (7/28/65), which results in an unclear set of results. From this we can say that there is only a possible trivial difference in treating a client with EA versus MA when looking at lower limb strength (Table 3).

\subsection{Effect size data summary}

The tables below show the results from Hopkins pre-post parallel group's spreadsheets. Table 1 represents the MAG versus the control group looking at average torque for the right and left ankles while performing plantar flexion. Table 2 represents the EAG versus control group looking at average torque for the right and left ankle while performing plantar flexion. The third table represents EA vs. manual acupuncture group looking at average torque for the right and left ankle while performing plantar flexion. The likelihood of this phenomenon happening is expressed as percentages as beneficial/trivial/detrimental. The clinical inference is based on the threshold chances of harmful $(0.5 \%)$ and beneficial $(25 \%)$.

Table 1. MAG versus control on average peak torque

\begin{tabular}{|c|c|c|c|c|}
\hline Average torque & Mean SD & Effect size SD & Likelihood & Clinical inference \\
\hline Plantar right & $31.4 \pm 34.2$ & $0.27 \pm 0.26$ & $69 / 31 / 0$ & Possible small positive effect $(+\mathrm{ve})$ \\
\hline Plantar left & $43.7 \pm 43.2$ & $0.51 \pm 0.42$ & $89 / 10 / 1$ & Very likely moderate + ve \\
\hline
\end{tabular}

Table 2. EAG versus control on average peak torque

\begin{tabular}{lcccc}
\hline Average torque & Mean SD & Effect size SD & Likelihood & Clinical inference \\
\hline Plantar right & $45.8 \pm 33.8$ & $0.4 \pm 0.24$ & $92 / 8 / 0$ & Likely small + ve \\
Plantar left & $66.1 \pm 53.8$ & $0.64 \pm 0.4$ & $96 / 4 / 0$ & Very likely moderate + ve \\
\hline
\end{tabular}

Table 3. EAG versus MAG on average peak torque

\begin{tabular}{lcccc}
\hline Average torque & Mean SD & Effect size SD & Likelihood & Clinical inference \\
\hline Plantar right & $-9.9 \pm 17.5$ & $-0.17 \pm 0.32$ & $3 / 52 / 44$ & Trivial \\
Plantar left & $-13.5 \pm 17.5$ & $-0.34 \pm 0.62$ & $7 / 28 / 65$ & Possibility + ve \\
\hline
\end{tabular}

\section{Discussion}

Acupuncture is a clinical intervention that is becoming more popular in sports medicine and exercise rehabilitation. A single session of acupuncture has been shown to be effective in enhancing muscle strength, although it was not significant ${ }^{[10]}$. However, when the number of treatment sessions was increased over a period of 4-6 weeks, the effects of acupuncture have been shown to significantly increase muscle strength $^{[11,14]}$. When looking purely at percentage change in torque values, the present study demonstrated that 18 sessions of bilateral needling at acupoints Zusanli (ST 36), Xiajuxu (ST 39) and Sanyinjiao (SP 6) resulted in a an average torque gain of $17 \%$ (right) and $8 \%$ (left) for manual acupuncture, 16\% (right) and $25 \%$ (left) for EA, and 6\% (right) and - 3\% (left) for the control group. The overall effect size for MA was small in the right limb with a likelihood of $69 \%$ that this result will take place every time. The effect size for the left limb was moderate with a likelihood of $89 \%$ that this result will take place every time. The overall effect size for the EAG was higher than that of the MAG, with a likelihood of $92 \%$ to $96 \%$ with a small effect in the right 
limb and medium effect size in the left limb. Our findings were similar to that of Huang LP, et a ${ }^{[11]}(21.3 \%$ right and $15.2 \%$ left), however, significantly lower than the findings of Zhou S, et al ${ }^{[14]}$ (range 35\%-64\% right, $32 \%-49 \%$ left compared to control group $-2 \%$ to + $2 \%)$. These differences can be explained due to the variation in methodologies. In our study we used the BIODEX which is the gold standard measurement in strength with far less chance in variability in recording data; furthermore, we looked at torque values rather than mass in kilogram. We took five measurements and calculated the average results, whereas Zhou S, et al ${ }^{[14]}$ took at least three measurements and then utilized the highest force value. While Huang LP, et al ${ }^{[11]}$ instructed participants to lift weights in $5 \mathrm{~kg}$ increments, this was continued until failure to complete dorsiflexion, then the last $5 \mathrm{~kg}$ weight was removed and a lighter weight was added with $1 \mathrm{~kg}$ increments. The final weight that was successfully lifted was recorded as the maximum dorsiflexion strength. Gandevia $\mathrm{SC}^{[30]}$ suggest that all maximum effort should be accompanied by some instruction and appropriate standardized verbal encouragement by the investigator during maximum voluntary contractions. In addition, subjects should be allowed to reject efforts that they do not regard as "maximal". No external motivation either verbal or visual was provided in this study during the pre- or post-test. However, these principles were employed by Zhou $S$, et $a l^{[14]}$ and would partly account for their results showing greater gain in strength in comparison to Huang LP, et $a^{[11]}$ and the current study. Our statistical method of using Hopkins spreadsheet is a much more valid way of looking at data rather than looking at pure percentage change of the raw data, however, we have included percentage data in the discussion to compare our results with other published data.

Overall, we found that both EA and MA presented with a greater effect to the intervention on the left (effect size medium) than on the right (effect size small) lower limb during ankle plantar flexion. We can hypothesize that this is due to a larger population being right side dominant thus the left side neural pathways have more potential to improve in strength and/or torque in regards to neural and hormonal adaptations as well as musculoskeletal. The small decrease found in the control group is unexplained, while it could be hypothesized as a result of participant motivation.

\subsection{Underlying mechanism of acupuncture in enhancing muscle strength}

In the majority of randomized controlled trials (RCTs) investigating the effects of acupuncture on enhancing athletic performance, Zusanli (ST 36) was employed ${ }^{[13]}$. However, with regards to the RCTs investigating the effect of acupuncture on enhancing muscle strength, only one study ${ }^{[9]}$ did not employ Zusanli (ST 36) and presented a negative effect of acupuncture on muscle strength. The majority of studies used transcutaneous electrical stimulation, which is suggested to activate motor nerve fibers, rather than directly activating muscle fibers, because the nerve fibers have higher excitability than muscle fibers. Huang $L P$, et al ${ }^{[11]}$ announced that their finding may have been a result of both acupoints Zusanli (ST 36) and Xiajuxu (ST 39), which are located above the deep peroneal nerve that innervates the ankle dorsi-flexors, and near the superficial peroneal nerve that has cutaneous branches to the anterior-lateral aspect of the leg. This may support the finding of Huang $L P$, et al ${ }^{[11]}$, Hubscher $\mathrm{M}$, et $a l^{[10]}$, and Zhou S, et $a^{[14]}$, which all assessed the effect of acupuncture on ankle dorsiflexion, however do not fully explain the underlying mechanism of the current study and that of other studies. So $\mathrm{RCH}$ and colleagues $^{[12]}$ assessed the effect of TENS at Zusanli (ST 36), Sanyinjiao (SP 6), Chengshan (BL 57) and Yanglingquan (GB 34) on enhancing rate of muscle force recovery after strenuous knee extension exercise. They found that TENS was significantly more effective than pseudo TENS in enhancing the rate of muscle extension peak torque force recovery. These points are not located in the quadriceps muscles or over the nerve trunk that supply these muscles.

In this study we assessed the effects of acupuncture on ankle plantar flexion strength using similar points [Zusanli (ST 36), Xiajuxu (ST 39) and Sanyinjiao (SP 6)] to Huang LP, et $a l^{[11]}$ and Zhou S, et $a l^{[14]}$. Sanyinjiao (SP 6) is located 3 cun above the prominence of the medial malleolus, posterior to the medial edge of the tibia. It is situated in the flexor digitorum longus, posterior tibialis and the flexor hallucis longus, which are all innervated by the tibial nerve. These muscles assist with ankle plantar flexion. Zusanli (ST 36) and Xiajuxu (ST 39) do not have a neural effect on the ankle plantar-flexors, however Zusanli (ST 36) has been shown by Gao M, et al ${ }^{[31-32]}$ and Toda $S^{[33-34]}$ to have a significant effect on general muscle function throughout the body that does not necessarily directly involve the central or peripheral nervous system in enhancing muscle function.

Carnitine is naturally synthesized in mammals from essential amino acids lysine and methionine ${ }^{[35]}$, and is primarily found in large volume in myocardial and skeletal muscle tissue. During energy metabolism, carnitine plays a major role in transporting long-chain fatty acid into mitochondrial matrix ${ }^{[31]}$, preserves the integrity of the mitochondrial membrane, reduces the production of lactate ${ }^{[36]}$, and allows the mitochondrial cells to utilize energy more efficiently. Carnitine and glutathione are antioxidants that have a protective role in reducing over production of free reactive oxygen species (ROS) within mitochondrial cells, which in turn reduce damage to muscle cell during intensive exercise. Strength exercises such as intensive muscle contraction 
produce typical reactive oxygen species generation ${ }^{[33,}$ 37-39] and will reduce the number of glutathione as it responds to the rise in ROS. Manual acupuncture and EA at Zusanli (ST 36) and Jiexi (ST 41) has been shown to significantly increase the volume of carnitine and glutathione in the circulation ${ }^{[32]}$ and muscles ${ }^{[29-30,33-34]}$ during intense exercise. An increase in carnitine and glutathione in the circulation and muscles following acupuncture would enhance the respiratory capacity of mitochondria in muscle fibers resulting in a slower utilization of muscle and blood glycogen, less lactate production during exercise and ability to oxidize pyruvate in response to intense training. Furthermore, it has been suggested that the accumulation of carnitine in the capillaries and endothelial cells may enhance oxygen delivery to exercising muscles thereby reducing muscle hypoxia normally observed during high-intensity exercise $^{[40]}$. This may explain some of the underlying effects of manual acupuncture at Zusanli (ST 36) during our study on enhancing muscle strength in the ankle plantar flexors.

Strength training forms an integral part of an athlete's preparation for competition, return to sport following injury or in the prevention of injuries. The findings from this study demonstrate the possible benefits of acupuncture during rehabilitation of musculoskeletal disorders, prevention of injuries and in the preparation of competitive athletes for competition.

\subsection{Limitations}

One of the limitations to the study, is that the participants all volunteered to take part in the study and some of which already had an interest in the topic of acupuncture, which may have resulted in participants wishing to give a positive response to the intervention. Another limitation to the study is that the activity levels of the participants could vary over the duration of the intervention. This was minimized by asking the participants not to alter their exercise levels over the duration of the intervention and by keeping a diary of their activity levels and activities of daily living to ensure that each week was same. A more substantial response from the intervention could have been elicited from a sedentary population. Furthermore, our results could have presented with a larger effect size and strength gain if we had followed the principles of Gandevia $\mathrm{SC}^{[30]}$. It is suggested that all maximum effort should be accompanied by some instruction and appropriate standardized verbal encouragement by the investigator during maximum voluntary contractions and subjects should be allowed to reject efforts that they do not regard as 'maximal ${ }^{[30]}$.

\section{Conclusion}

Our study demonstrated that EA and MA significantly improve plantar flexion muscle strength compared with the control group which showed a slight reduction in muscle strength in healthy subjects which could be hypothesized that the slight reduction is due to participant motivation.

\section{Conflict of Interest}

The authors declared that there was no potential conflict of interest in this article.

Statement of Informed Consent

Informed consent was obtained from all individual participants included in this study.

Received: 31 July 2016/Accepted: 28 August 2016

\section{References}

[1] Furlan AD, van Tulder MW, Cherkin DC, Tsukayama H, Lao L, Koes BW, Berman BM. Acupuncture and dry-needling for low back pain. The Cochrane Database of Systematic Reviews Cochrane Database Syst Rev, 2005, 25(1): 10-11.

[2] Ernest E, Lee MS, Cho TY. Acupuncture: does it alleviate pain and are there serious risks? A review of reviews. Pain, 2011, 152(4): 755-764

[3] Akimoto T, Nakahori C, Aizawa K, Kimura F, Fukubayashi T, Kono I. Acupuncture and responses of immunologic and endocrine markers during competition. Med Sci Sports Exerc, 2003, 35(8): 1296-1302.

[4] Gentil D, Assumpcao J, Yamamura Y, Barros NT. The effect of acupuncture and moxibustion on physical performance by sedentary subjects submitted to ergospirometric test on the treadmill. J Sports Med Phys Fitness, 2005, 45(1): 134-140.

[5] Lin ZP, Lan LW, He TY, Lin SP, Lin JG, Jang TR, Ho TJ. Effects of acupuncture stimulation on recovery ability of male elite basketball athletes. Am J Chin Med, 2009, 37(3): 471481.

[6] Ehrlich D, Haber P. Influence of acupuncture on physical performance capacity and haemoydynamic parameters. Int $\mathbf{J}$ Sports Med, 1992, 13(6): 486-491.

[7] Cheung LC, Jones AY. Effect of Acu-TENS on recovery heart rate after treadmill running exercise in subjects with normal health. Complement Ther Med, 2007, 15(2): 109-114.

[8] Itoh K, Ochi H, Kitakoji H. Effects of tender point acupuncture on delayed onset muscle soreness (DOMS): a pragmatic trial. Clin Med, 2008, 3(1): 14.

[9] Hubscher M, Vogt L, Bernhorster M, RosenHagen A, Banzer W. Effects of acupuncture on symptoms and muscle function in delayed-onset muscle soreness. J Altern Complement Med, 2008, 14(8): 1011-1016.

[10] Hubsher M, Vogt L, Ziebart T, Banzer W. Immediate effects of acupuncture on strength performance: a randomized crossover trial. Eur J Applied Physiology, 2010, 110(2): 353358.

[11] Huang LP, Zhou S, Lu Z, Tian Q, Li X, Cao LJ, Yu JH, Wang $H$. Bilateral effect of unilateral electroacupuncture on muscle strength. J Altern Complement Med, 2007, 13(5): 539-546.

[12] So RCH, Ng JKF, Ng GYF. Effect of transcutaneous electrical acupoint stimulation on fatigue recovery of the quadriceps. Eur J Applied Physiology, 2007, 100(6): 693-700.

[13] Bailey SD. Effects of acupuncture on human performance: a 
systematic review. J AACP, 2012: 23-42.

[14] Zhou S, Huang LP, Liu J, Yu JH, Tian Q, Cao LJ. Bilateral effects of 6 weeks' unilateral acupuncture and electroacupuncture on ankle dorsiflexors muscle strength: a pilot study. Arch Phys Med Rehabil, 2012, 93(1): 50-55.

[15] Maioli C, Falciati L, Marangon M, Perini S, Losio A. Shortand long-term modulation of upper limb motor-evoked potentials induced by acupuncture. Eur J Neurosci, 2006, 23(7): 1931-1938.

[16] Hopkins WG, Marshall SW, Batterham AM, Hanin J. Progressive statistics for studies in sports medicine and exercise science. Med Sci Sports Exerc, 2009, 41(1): 3-13.

[17] Holmbäck AM, Porter MM, Downham D, Lexell J. Reliability of isokinetic ankle dorsiflexor strength measurements in healthy young men and women. Scand J Rehabil Med, 1999, 31(4): 229-239.

[18] Foran B. High-performance Sports Conditioning. Windsor: Human Kinetics Publishers, 2001: 169.

[19] Goubel F, Lensel-Corbeil G. Biomécanique. Elements of Muscular Mechanics. Paris: Editions Masson, 2003.

[20] Hauraix H, Nordez A, Dorel S. Shortening behavior of the different components of muscle-tendon unit during isokinetic plantar flexions. J Appl Physiol, 2013, 115(7): 1015-1024.

[21] Jarmey C, Bouratnos I. A Practical Guide to Acu-points. West Sussex: Lotus Publishing, 2008: 221-222.

[22] Cross JR. Acupressure, Clinical Applications in Musculoskeletal Conditions. Oxford: Butterworth-Heinmann, 2000: 23.

[23] Maffiuletti NA, Cometti G, Amiridis IG, Martin A, Pousson M, Chatard JC. The effects of electromyostimulation training and basketball practice on muscle strength and jumping ability. Int J Sports Med, 2000, 21(6): 437-443.

[24] Blümel G. Electromyostimulation in Traumatology. Stuttgart: Georg Thieme Verlag, 1992: 21-26.

[25] Cohen J. Statistical power analysis. Current Directions in Psychological Science, 1992, 1(3): 98-101.

[26] Hopkins WG. Sample sizes for magnitude-based inferences about clinical, practical or mechanistic significance. Med Sci Sport Exer, 2006, 38: S528-S529.

[27] Vickers AJ, Altman DG. Statistics notes: analyzing controlled trials with baseline and follow up measurements. BMJ, 2001, 323(7321): 1123-1124.

[28] Batterham A M, Hopkins WG. Making meaningful inferences about magnitudes. Int J Sports Physiol Perform, 2006, 1(1): 50-57.

[29] Kirk RE. Practical significance: a concept whose time has come. Educ Psychol Meas, 1996, 56(5): 746-759.

[30] Gandevia SC. Spinal and supraspinal factors in human muscle fatigue. Physiol Rev, 2001, 81(4): 725-789.

[31] Gao M, Yang HY, Liu TY, Kuai L. Effects of manual acupuncture and electroacupuncture on mitochondria of skeletal muscle cells in rats of acute swimming exercise. Zhongguo Zhen Jiu, 2005, 25(6): 421-424.

[32] Gao M, Yang HY, Le K, Liu TY, Gu XJ. Effects of manual acupuncture and electroacupuncture on $\mathrm{Ca}^{2+}$ content and $\mathrm{Ca}^{2+}$-ATPase activity in sarcoplasmic reticulum of skeletal muscle cells in rats during acute swimming exercise. Zhen $\mathrm{Ci}$ Yan Jiu, 2008, 33(1): 13-16.

[33] Toda S. Investigation of electroacupuncture and manual acupuncture on carnitine and glutathione in muscle. Evid Based Complement Alternat Med, 2011: 297130.

[34] Toda S. Effects of electroacupuncture on carnitine in serum and brain. World J Acupunct Moxibustion, 2007, 17(2):22-24.

[35] Bremer J. Carnitine metabolism and functions. Physiol Rev, 1983, 63(4):1420-1480.

[36] Siliprandi N, Di Lisa F, Menabo R. Clinical use of carnitine. Past, present and future. Adv Exp Med Biol, 1990, 272: 175-181.

[37] Corbucci GG, Montanari G, Mancinelli G, D’Iddio S. Metabolic effects induced by L-carnitine and propionyl-Lcarnitine in human hypoxic muscle tissue damage during exercise. Int J Clinical Pharmacology Research, 1990, 10(3): 197-202.

[38] Giamberadino MA, Dragani L, Valente R, Lisa FD, Saggini R, Vecchiet L. Effects of prolonged L-carnitine administration on delayed muscle pain and CK release after eccentric effort. Int J Sports Medicine, 1996, 17: 320-324.

[39] Volek JS, Kraemer WJ, Rubin MR, Gomez AL, Ratamess NA, Gaynor P. L-carnitine L-tartrate supplementation favorably affects markers of recovery from exercise stress. Am J Physiol Endocrinal Metabolic, 2002, 282(2): E474-482.

[40] Kraemer WJ, Volek JS, French DN, Rubin MR, Sharman MJ, Gomez AL, Ratamess NA, Newton RU, Jemiolo B, Craig BW, Häkkinen K. Effects of L-Carnitine L-Tartrate supplementation and hormonal response to resistance exercise and recovery. J Strength Cond Res, 2003, 17(3): 455-462. 